This is the accepted manuscript of the article, which has been published in Heart. 2018, 104(9), 773-778. http://dx.doi.org/10.1136/heartjnl-2017-312136

\title{
Aortic sinus diameter in middle age is associated with body size in young adulthood
}

Jussi A. Hernesniemi ${ }^{1,2}$, Jarkko Heiskanen ${ }^{3}$, Saku Ruohonen $^{3}$, Noora Kartiosuo ${ }^{3}$, Nina Hutri-Kähönen ${ }^{4}$, Mika Kähönen ${ }^{5}$, Eero Jokinen ${ }^{6}$, Päivi Tossavainen ${ }^{7}$, Merja Kallio ${ }^{7}$, Tomi Laitinen ${ }^{8}$, Terho Lehtimäki ${ }^{2}$, Jorma Viikari ${ }^{9,10}$, Markus Juonala ${ }^{9,10}$, and Olli Raitakari ${ }^{3,11}$

${ }^{1}$ Heart Hospital, Tampere University Hospital and Faculty of Medicine and Life Sciences, University of Tampere, Tampere, Finland. ${ }^{2}$ Department of Clinical Chemistry, Fimlab Laboratories, and Finnish Cardiovascular Research Center Tampere, Faculty of Medicine and Life Sciences, University of Tampere, Tampere, Finland. ${ }^{3}$ Research Center of Applied and Preventive Cardiovascular Medicine, University of Turku, Turku, Finland. ${ }^{4}$ Department of Pediatrics University of Tampere and Tampere University Hospital. ${ }^{5}$ Department of Clinical Physiology, Tampere University Hospital, University of Tampere, Tampere, Finland. ${ }^{6}$ Children's Hospital University, University Central Hospital Helsinki, Helsinki, Finland. ${ }^{7}$ Department of Pediatrics, University of Oulu, Oulu, Finland; PEDEGO Research Unit, and Medical Research Center Oulu, University of Oulu, Oulu, Finland. ${ }^{8}$ Department of Clinical Physiology, Kuopio University Hospital, University of Eastern Finland, Kuopio, Finland. ${ }^{9}$ Department of Medicine, University of Turku, Turku, Finland. ${ }^{10}$ Division of Medicine, Turku University Hospital, Turku, Finland. ${ }^{11}$ Department of Clinical Physiology and Nuclear Medicine, Turku University Hospital, Turku, Finland.

Word count: 2994

Adress of Correspondence: Jussi Hernesniemi, MD, PhD, University of Tampere, Tampere, Finland Address: Finn-Medi 1 (2nd floor), Biokatu 6, 33521 Tampere, Finland e-mail: Jussi.hernesniemi@uta.fi_Tel: +358504306051 


\section{Abstract}

Objective-Aortic sinus dilatation can lead to aortic valve regurgitation or even aortic dissection. Our objective was to examine the association between body surface area (BSA) measures from childhood to middle age and aortic sinus diameter in middle age. Understanding the relation of these two clarifies how aortic size is normally determined.

Methods - Cardiovascular Risk in Young Finns Study is a longitudinal study with follow-up of over 31 years (1980-2011). The study comprises information of body composition from multiple time points of 1,950 subjects with cardiac ultrasound measurements made in 2011. The association between BSA in different ages and aortic sinus diameter in middle age was analyzed by linear regression modeling adjusted with age, sex and diastolic blood pressure. Missing BSA values were derived for each life year (ages 3-33) from subject-specific curves for body weight and height estimated from longitudinal measurements using mixed model regression splines.

Results - BSA estimates in early twenties are most strongly associated with aortic sinus diameter in middle age. Top association was observed at age 23 years with one SD increase in estimated BSA corresponding to $1.04 \mathrm{~mm}(0.87-1.21 \mathrm{~mm})$ increase in aortic diameter. Increase in body weight beyond early twenties does not associate with aortic sinus diameter and the association between middle age BSA and aortic size is substantially weaker $(0.74 \mathrm{~mm}$ increase $[0.58-0.89 \mathrm{~mm}])$. These results were confirmed in a subpopulation using only measured data.

Conclusion-The association between aortic sinus diameter and BSA is stronger when considering BSA in young-adulthood compared to BSA in middle age.

Keywords: Ascending aorta, Body size, Dilatation, Echocardiography 


\section{Key questions}

\section{What is already known about the subject?}

Body surface area (BSA) along with age and sex is one of the most significant determinants of aortic size measured at sinuses of Valsalva. Indexing aorta diameter to BSA can be used to evaluate normalcy in clinical work but previous research suggests that the association between aortic sinus diameter and BSA diminishes by age. This could affect negatively the evaluation of relative aortic size in older ages.

\section{What does this study add?}

This study shows that aortic sinus diameter is most strongly associated with BSA measured in early twenties. Subsequent changes in body weight (usually resulting in higher BSA by developing obesity) do not associate with aortic sinus diameter.

\section{How might this add to clinical practice?}

The result of the present study suggests that BSA measured in the early twenties may be the best measure of body size to use when evaluating the aortic sinus diameter relative to body size. However, more research is needed to confirm that using early-life body size leads to better clinical outcomes. 


\section{INTRODUCTION}

Dilatation of the proximal aorta, most often observed at Sinuses of Valsalva, can lead to aortic valve regurgitation and to aortic dissection even without coexisting aortic valve pathology or genetic connective tissue disorder [1-3]. Normally sex, age and body size estimated by body surface area (BSA) are the most significant determinants of aortic sinus diameter. According to current American and European guidelines an aortic size of approximately $1.9-2.0 \mathrm{~cm} / \mathrm{m}^{2}$ indexed to BSA may be considered the upper limit of normal aortic size measured at sinuses of Valsalva $[1,3,4]$.

Previous studies have shown that obesity and increased body height in childhood associate with increased left ventricular size and abdominal aortic size [5,6]. As the circulatory system develops parallel with body size during early life, it is plausible that the aortic sinus size of is also determined by body size in early life. Supporting this, aortic sinus size is strongly correlated with BSA among children but only moderately among middle aged population [7]. Most often aortic dilatation is suspected incidentally when performing transthoracic ultrasound in middle age or later. By then body weight has usually changed significantly from early life possibly interfering with the evaluation of normal aortic size in relation to BSA.

The purpose of this study is to analyze the effect of developing body size from childhood to middle age on the size of aortic sinuses measured in early middle age. Understanding the relation of these two can clarify the underlying mechanisms of aortic growth and help define the limits of normal aortic size. To address this question, we used the longitudinal data and cardiac ultrasound measurements of the ongoing Cardiovascular Risk in Young Finns Study [8]. 


\section{METHODS}

\section{Study cohort}

The Cardiovascular Risk in Young Finns Study starter in 1980 with random recruitment of six age cohorts $(3,6,9,12,15$ and 18 years) from five predefined geographical areas using data from the national registry. Altogether 3,596 (83\%) of the 4,320 invitees attended. Subsequent follow-ups were performed in three-year intervals until 1992, and additional follow-ups were organized in 2001, 2007 and 2011 [9] resulting in 31 years of follow-up. In 2011, transthoracic ultrasound was performed on 1,994 subjects. For the purpose of this study, full data set of aortic measurements with complete follow-up data of the studied factors is available from 1,950 subjects. A flow chart of the study including the measured factors in each follow-up is presented in Figure 1. Participants who were lost to follow-up between 1980 and 2011 were more often men than women $(51 \%$ vs. 43\%, p<0.001) but there were no statistically significant $(\mathrm{p}<0.05)$ differences in baseline age, body mass index (BMI) or blood pressure values among men or women. During the follow-up, 93 of the original participants died with only $14.0 \%(n=13)$ of the deaths attributable to cardiovascular causes (none to aortic or valvular causes). Specific details of the methodology have been described earlier [8,9]. All study participants or their legal guardians if the participant was under 18 year of age, gave their written informed consent. Local ethics committees at all recruiting study centres have approved the study protocol

\section{Cardiac ultrasound}

Transthoracic echocardiography was performed by a sonographer with Acuson Sequoia 512 (Acuson, Mountain View, CA) ultrasonography, using $3.5 \mathrm{MHz}$ scanning frequency phased-array transducer at each study center. Aortic dimensions were obtained using standard M-mode images measuring the 
distances from leading edge-to-edge diameter in end-diastole from long axis view. The analysis of the images was done by one observer using ComPACS 10.7.8 analysis program (MediMatic Solutions, Genova Italy). Aortic sinus diameter was measured from sinuses of Valsalva which most commonly also provides largest measurement for absolute aortic diameter. Images were also used to obtain measurement from other sites of the thoracic aorta (aortic annulus, sinotubular junction and tubular section) and to verify the morphology of the ascending aorta. As expected, with the exception of only four study participants, the measurements from sinuses of Valsalva provided largest diameter values when compared to aortic annulus or sinotubular junction. The primary purpose of the study was not to identify subjects with bicuspid valves and therefore all subjects who attended were included to the study. The methodology for the imaging has been described earlier in more detail [10].

\section{Statistical analysis}

The main outcome variable was aortic sinus diameter measured in middle age (between ages 34-49 years). Main exposure variable depicting body size was body size indexed to body surface area (BSA) by body weight and height using the formula by Dubois\&Dubois where BSA is determined as BSA= $0.007184 \mathrm{x}$ height $(\mathrm{m})^{0.725} \mathrm{x}$ weight $(\mathrm{kg})^{0.425}[11]$.

Body height and weight were measured at the follow-up visits from age points 3, 6, 9, 12, 15, and 18 in year 1980 to age points 34, 37, 40, 43, 46, 49 in year 2011 for each study subject participating the follow-ups. Due to the study design (separate age cohorts with three year age intervals) and nonparticipation of all original participants in all follow-ups, information for all ages from all participants was not available. For this reason, yearly estimates of height and weight during early life years (ages 3- 
34) were obtained (estimated) by fitting subject-specific curves as mixed model regression splines. For example, the height $Y_{i j}$ of individual at time $t_{i j}$ was modelled as follows:

$Y_{i j}=\left[\alpha+\beta t_{i j}+\sum_{k=1}^{5} \gamma_{k} B_{k}\left(t_{i j}\right)\right]+\left[a+b_{i} t_{i j}+\sum_{l=1}^{3} c_{i l} B_{l}\left(v_{i j}\right)\right]+e_{i j}$,

The model entails a population average profile as the fixed part (the terms in the first brackets) as well as the subject-specific deviation from the mean profile as the random part (the terms in the second brackets), and the measurement error term $e_{i j}$. In addition to the linear parts in the model, the spline terms are included to model the possible nonlinear trends in the population average profile and the subject-specific profiles. The term $v_{i j}$ in the random spline term is the duration of the follow-up from the baseline visit. The B-functions in spline terms are basis functions, which were used for constructing the splines. Corresponding model was used for weight.

To avoid over-fitting, the number of knots in the subject-specific part was reduced (two knots on the calendar time from 1980 to 2011) from that of the fixed effects part (four knots on age from 3 to 34 years). The population average profile was allowed to vary across birth cohorts and sex.

Using splines provides a very accurate prediction of subjects weight and height for missing data points [12]. As expected, the resulting correlation between thus estimated and actually measured data for BSA is strong as age dependent development of height and weight is very consistent. For age groups with applicable data for comparison, the resulting correlation coefficients for BSA values ranged between 0.978-0.992 (Age groups: 3 [n=265], 6 [ $n=548], 9$ [ $n=866$ ], 12 [n=926], 15 [n=945], 18 [ $\mathrm{n}=968], 21[\mathrm{n}=560], 24[\mathrm{n}=543], 27[\mathrm{n}=346], 30[\mathrm{n}=563]$ and 33 [n=529] years). The results obtained using the estimated values in the entire study population were further confirmed by using only this measured follow-up data. 
Multivariable linear regression analysis was used to study the association between estimated and BSA in different ages and aortic sinus diameter measured in middle age. In order to compare the relative strengths of the associations, all BSA values were age standardized and centered to mean (ztransformed with mean=0 and $\mathrm{SD}=1$ ). All analyses were adjusted for age, sex and diastolic blood pressure which are the most significant factors associating with aortic sinus diameter ( $\mathrm{p} \leq 0.001$ for all). 


\section{RESULTS}

\section{Population characteristics}

Characteristics of study population measured in 2011 are presented in Table 1 . The study population comprises relatively healthy Caucasian general population. At baseline (in 1980) when most of the subjects were still children and adolescents, only $0.7 \%$ of the subjects were obese (age and sex specific values corresponding to adult $\mathrm{BMI} \geq 30 \mathrm{~kg} / \mathrm{m}^{2}$ ), none had type 2 diabetes and $13.1 \%$ were daily smokers. The development of BSA in the study population in early life is illustrated in Figure 2.

Mean aortic sinus diameter was $3.44 \mathrm{~cm}(\mathrm{SD} 0.30)$ or $1.68 \mathrm{~cm} / \mathrm{m}^{2}(\mathrm{SD} 0.18)$ for men and $3.02 \mathrm{~cm}$ (SD 0.26 ) and $1.70 \mathrm{~cm} / \mathrm{m}^{2}$ (SD 0.19) for women. In the entire study population one-year increase in age associated with $0.10 \mathrm{~mm}$ (95\% CI: 0.08-0.13) increase in aortic sinus diameter. Overall, 107 subjects $(5.0 \%)$ had BSA indexed aortic sinus diameter greater than $2.0 \mathrm{~cm} / \mathrm{m}^{2}$. Sixteen of these subjects $(15 \%)$ had an aortic sinus diameter of $4.0 \mathrm{~cm}$ or greater (only one female). Altogether forty-one subjects had aortic size of $4.0 \mathrm{~cm}$ or greater representing $2.1 \%$ of the population.

\section{Associations of BSA measured in different ages with ascending aortic diameter in middle-aged}

By observing adjusted regression coefficients, aortic sinus diameter measured in middle age associated most strongly with estimated BSA values in early twenties (between ages 20-25 years)(Figure 3). The association weakened gradually after that age (Figure 3). This age corresponds to the age when the rapid growth of BSA is stalled by the end of body height growth and declining rate of lean mass development (Figure 2). After early twenties BSA still grows but to a much slower pace and the two figures no longer convergence (Figure 2 and 3). The highest regression coefficient for the association 
was observed for estimated BSA at the age of 23 years (coefficient 1.04 with 95\% CI 0.87-1.21). The regression coefficient for BSA measured at the time of the ultrasound (in middle age) was significantly weaker (coefficient 0.74 with $95 \%$ CI 0.58-0.89). This means that one SD change in estimated BSA at age of 23 years associates with approximately $1.04 \mathrm{~mm}$ change in aortic sinus diameter whereas one SD change in BSA in middle age associates with a $0.74 \mathrm{~mm}$ change. In absolute terms this means that a 0.1 unit change in BSA at the age of 23 years corresponds to a $0.53 \mathrm{~mm}$ difference in observed aortic size as opposed to a $0.33 \mathrm{~mm}$ difference when BSA is measured in middle age.

In order to control for any possible inaccuracies related to the estimated BSA data, the results were confirmed using only observed data by applying linear mixed modeling with repeated measures with an interaction between age at each BSA measurement and observed BSA thus allowing the association between BSA and aortic sinus diameter to be different at different ages. In line, the highest correlation coefficient was seen between BSA observed at the age of 21 years and middle age aortic sinus diameter (coefficient 0.086 with $95 \%$ CI 0.064-0.109) after which the strength of the association declined. Finally, when focusing on subjects with observed data at the age of 21 years and at the time of the ultrasound (an age cohort, that also presented with the absolute highest correlation coefficient in linear regression analysis, $n=556$ ), the difference in correlation coefficients for was substantial (coefficient 1.45 with $95 \%$ CI $0.11-1.76$ for BSA at age 21 vs. 0.95 with $95 \%$ CI $0.66-1.24$ in middle age) supporting the findings obtained using estimated values.

For clinical reference, the mean aortic sinus diameter in middle age indexed to BSA in early twenties was $1.77 \mathrm{~cm} / \mathrm{m}^{2}$ for men and $1.81 \mathrm{~cm} / \mathrm{m}^{2}$ for women. The normal upper limits defined by $95 \%$ threshold for observed values were $2.10 \mathrm{~cm} / \mathrm{m}^{2}$ for men and $2.12 \mathrm{~cm} / \mathrm{m}^{2}$ for women. The association between BSA estimated in early twenties (at age 23) and aortic sinus diameter measured in middle age (between ages 34-49) in men and women is presented in Figure 4. 


\section{The impact of weight change from early adulthood to middle age on aortic size}

The fact that aortic size is most strongly associated with BSA measured in early adulthood suggests that aortic size is determined by body size developed up until that point. We analyzed the association of subsequent changes ( $\Delta$-values) in body weight (and BSA) from that age period to middle age. As individual height remains almost constant from early adulthood to early middle age, the changes in BSA only reflect body weight gain or loss between these ages. According to linear regression analysis (model also adjusted with average of BSA between ages 21-25), $\Delta$-weight did not associate significantly with aortic sinus diameter (10kg change corresponding to a $-0.065 \mathrm{~mm}$ change in diameter with $95 \%$ CI -0.186 to $0.056, \mathrm{p}=0.290)$. The associations between change in body weight and aortic sinus dimension were not significantly different among men and females ( $p>0.4$ for interaction) and the associations remained constant regardless of the baseline BSA ( $p>0.9$ for interaction). Furthermore, there was no significant interaction with age (cohort) showing that the effect of weight change is not significantly different regardless the time in which it occurs ( $p>0.4$ for interactions) as the time difference between early adulthood and the ultrasound study in middle age up were approximately 10 years for the youngest age cohort and 25 years for the oldest age cohort. 


\section{DISCUSSION}

The novel results of this study reveal that compared with BSA measurements from other ages, BSA in early twenties associates strongest with aortic sinus diameter in middle age. Weight gain after early adulthood does not seem to have substantial impact on aortic size. This age (early twenties) marks also the age when the rapid growth of BSA reaches its plateau suggesting that aortic size at sinuses of Valsalva is strongly determined by body size development until that point.

The major indication of the present results is that estimating the relation between body size and aortic sinus diameter, using contemporary BSA in middle age can lead into underestimating the relative size of aortic size if the subject has substantially gained weight after early adulthood. Obviously, this finding does not impact middle aged (or older) subjects who already have aortic sinus size larger than normal by current standards (indexed to BSA or exceeding the absolute limit of 40mm) who should be monitored and treated according to established guidelines [1,3,4]. However, middle-aged subjects who are found to have relative aortic sinus size below but near the upper limit of normal and who have substantially gained weight after early adulthood, should probably be monitored for possible abnormal growth. For example, if a male subject with BSA of $1.7 \mathrm{~m}^{2}$ in early adulthood gains $30 \mathrm{~kg}$ (or $66 \mathrm{lbs}$ ) when becoming middle aged (corresponding to $\sim 0.3 \mathrm{~m}^{2}$ change in BSA), he is considered to have a normal relative aortic size measured at $38 \mathrm{~mm}$ in middle age (corresponding to $1.9 \mathrm{~cm} / \mathrm{m}^{2}$ indexed to BSA). In reality, the relative size of the aorta could be already abnormal. As changes in body weight seem to have very little impact of aortic diameter in later life, the possible abnormal growth tendency should be reliably distinguished by recurrent transthoracic ultrasound follow-ups according to established guidelines [1,3]. In line, dedicated longitudinal studies in adults over the age of 30 years of age have shown that aortic sinus size increases very little with increasing bodyweight $[13,14]$. In the Framingham risk study in a population with a mean age of 52 years, 10 -unit increase in BMI (23-40kg 
increase between subjects of $150 \mathrm{~cm}$ and $200 \mathrm{~cm}$ of height) in a time period of 16 years corresponds to only approximately $1 \mathrm{~mm}$ change in aortic diameter [13]. Correspondingly results of the Coronary Artery Risk Development in Young Adults Study with mean age of 30 years at the beginning of the study have shown that a increase of $10 \mathrm{~kg}$ in body mass over a 20 year period associates with 1.1 $1.2 \mathrm{~mm}$ increase in aortic diameter [14].

In order to avoid confounding by overweight, it has been also proposed that aortic diameter be indexed to height instead of BSA. However, existing guidelines recommend using BSA because evidence of large series for height indexed values are lacking and BSA seems superior to height in explaining aortic size $[1,3,4,15]$. In the present study, we did not compare the predictive value of height and BSA but indexing aortic size to BSA in early twenties accounts for the confounding by middle age overweight and obesity but still captures the possible residual effect of weight gains during at the end of puberty. In line with current recommendations the upper limit for aortic size index to BSA is similar in both sexes (approximately $2.1 \mathrm{~cm} / \mathrm{m}^{2}$ in our population). The limitation of our data is that as aortic size increases approximately $0.7-1 \mathrm{~mm}$ for each decade for life, our reference figures should be interpreted with caution in older patients.

Observing that that aortic size measured at sinuses of Valsalva is most strongly predicted by body size in early life leads to the question of the relative impact of body weight and the possible effect of early life obesity. Previous studies have showed that body weight seems to a have smaller relative impact on aortic sinuses than body height $[14,16]$. Therefore it is questionable whether preventing childhood obesity could prevent aortic sinus dilatation which is a strong risk factor for aortic regurgitation even in the absence of abnormal valve morphology [17-19]. At least among adults, high BMI is not a substantial risk factor for developing aortic regurgitation or aortic dissection $[2,20,21]$ 
The strengths of the present study are the high quality follow-up data of cardiovascular risk factors from childhood to middle age including anthropometric and physiological factors over the course of 31 years. During the follow-up time there has been only minimal loss to follow-up due to cardiovascular mortality. The homogenous study population facilitates the observation of the impact of risk factors on the studied endpoints.

Main limitation of the present study is that we do not have ultrasound measurements of aortic sinuses from different age periods before middle age. Multiple measurements would provide reliable measurements for the development of aortic size with age in respect to changes in body size. However, given the very modest magnitude of the association between aortic growth tendency and changes in body weight in previous studies, our main conclusions seem justified. Additionally, as we did not screen for or excluded subjects with possible valvular or aortic abnormalities (which are rare), the main findings are mainly based on observations of general population and not applicable to specific and rare patient groups such as subjects with bicuspid aortic valve or other aortic pathology.

In conclusion, aortic sinus diameter seems to be mainly determined by achieved body size in early twenties. Weight gain after that age does not have substantial impact on aortic size. 


\section{ACKNOWLEDGEMENTS}

The Young Finns Study has been financially supported by the Academy of Finland: grants 286284 (T.L.), 134309 (Eye), 126925, 121584, 124282, 129378 (Salve), 117787 (Gendi), and 41071 (Skidi); the Social Insurance Institution of Finland; Competitive State Research Financing of the Expert Responsibility areas of Kuopio, Tampere and Turku University Hospital Medical Funds (grant X51001 for T.L.); Juho Vainio Foundation; Paavo Nurmi Foundation; Finnish Foundation of Cardiovascular Research; Finnish Cultural Foundation; Tampere Tuberculosis Foundation; Emil Aaltonen Foundation; Yrjö Jahnsson Foundation; Signe and Ane Gyllenberg Foundation and Diabetes Research Foundation of Finnish Diabetes Association. The authors have no relationship with industry resulting in possible conflicts of interest.

\section{CONTRIBUTORSHIP}

JAH, SR, TeL, OR and JV planned the present study. JAH, JH, SR, NK, N-HK, EJ, PT, MK, ToL, TeL, MJ conducted the study. All authors (JAH, JH, SR, NK, N-HK, MK, EJ, PT, MK, ToL, TeL, JV, MJ and OR) have been involved in reporting the study (i.e.drafting the work or revising it critically for important intellectual content). Jussi A. Hernesniemi is responsible for the data analysis and along with Olli Raitakari is responsible of the overall content of the work. All authors have given their final approval for the manuscript.

\section{FUNDING STATEMENT}

The funders of this study (as listed in acknowledgements) have had no part in designing or conduction of this study. 


\section{COMPETING INTERESTS}

None.

ETHICS APPROVAL

Local ethics committees at all recruiting study centres have approved the study protocol.

\section{AUTHOR STATEMENT}

The Corresponding Author has the right to grant on behalf of all authors and does grant on behalf of all authors, an exclusive licence (or non exclusive for government employees) on a worldwide basis to the BMJ Publishing Group Ltd and its Licensees to permit this article (if accepted) to be published in HEART editions and any other BMJPGL products to exploit all subsidiary rights. 


\section{REFERENCES}

1 Hiratzka LF, Bakris GL, Beckman JA, et al. 2010

ACCF/AHA/AATS/ACR/ASA/SCA/SCAI/SIR/STS/SVM guidelines for the diagnosis and management of patients with thoracic aortic disease: executive summary. A report of the American College of Cardiology Foundation/American Heart Association Task Force on Practice Guidelines, American Association for Thoracic Surgery, American College of Radiology, American Stroke Association, Society of Cardiovascular Anesthesiologists, Society for Cardiovascular Angiography and Interventions, Society of Interventional Radiology, Society of Thoracic Surgeons, and Society for Vascular Medicine. Catheter Cardiovasc Interv. 2010;76:E4386.

2 Braverman AC. Acute aortic dissection: clinician update. Circulation 2010;122:184-8. doi:10.1161/CIRCULATIONAHA.110.958975

3 Erbel R, Aboyans V, Boileau C, et al. 2014 ESC Guidelines on the diagnosis and treatment of aortic diseases: Document covering acute and chronic aortic diseases of the thoracic and abdominal aorta of the adult. The Task Force for the Diagnosis and Treatment of Aortic Diseases of the European Society of Cardiology (ESC). Eur. Heart J. 2014;35:2873-926. doi:10.1093/eurheartj/ehu281

4 Evangelista A, Flachskampf FA, Erbel R, et al. Echocardiography in aortic diseases: EAE recommendations for clinical practice. Eur J Echocardiogr 2010;11:645-58.

doi:10.1093/ejechocard/jeq056

5 de Jonge LL, de Jonge LL, van Osch-Gevers L, et al. Growth, Obesity, and Cardiac Structures in Early Childhood: The Generation R Study. Hypertension 2011;57:934-40.

doi:10.1161/HYPERTENSIONAHA.110.163303

6 Ciccone MM, Miniello V, Marchioli R, et al. Morphological and functional vascular changes induced by childhood obesity. European Journal of Cardiovascular Prevention \& Rehabilitation 2011;18:831-5. doi:10.1177/1741826711398180

7 Roman MJ, Devereux RB, Kramer-Fox R, et al. Two-dimensional echocardiographic aortic root dimensions in normal children and adults. AJC 1989;64:507-12.

8 Raitakari OT, Juonala M, Rönnemaa T, et al. Cohort profile: the cardiovascular risk in Young Finns Study. International journal of epidemiology 2008;37:1220-6. doi:10.1093/ije/dym225

9 Raitakari OT, Juonala M, Kähönen M, et al. Cardiovascular risk factors in childhood and carotid artery intima-media thickness in adulthood: the Cardiovascular Risk in Young Finns Study. JAMA 2003;290:2277-83.

10 Ruohonen S, Koskenvuo JW, Wendelin-Saarenhovi M, et al. Reference Values for Echocardiography in Middle-Aged Population: The Cardiovascular Risk in Young Finns Study. Echocardiography (Mount Kisco, NY 2015;:n/a-n/a. doi:10.1111/echo.13025 
11 Bois Du D, Bois Du EF. A formula to estimate the approximate surface area if height and weight be known. 1916. 1989.

12 Welham SJ, Cullis BR, Kenward MG, et al. The analysis of longitudinal data using mixed model L-splines. Biometrics 2006;62:392-401. doi:10.1111/j.1541-0420.2005.00500.x

13 Lam CSP, Xanthakis V, Sullivan LM, et al. Aortic root remodeling over the adult life course: longitudinal data from the Framingham Heart Study. Circulation 2010;122:884-90. doi:10.1161/CIRCULATIONAHA.110.937839

14 Teixido-Tura G, Almeida ALC, Choi E-Y, et al. Determinants of Aortic Root Dilatation and Reference Values Among Young Adults Over a 20-Year Period: Coronary Artery Risk Development in Young Adults Study. Hypertension 2015;66:23-9. doi:10.1161/HYPERTENSIONAHA.115.05156

15 Vasan RS, Larson MG, Benjamin EJ, et al. Echocardiographic reference values for aortic root size: the Framingham Heart Study. J Am Soc Echocardiogr 1995;8:793-800.

16 Davis AE, Lewandowski AJ, Holloway CJ, et al. Observational study of regional aortic size referenced to body size: production of a cardiovascular magnetic resonance nomogram. $J$ Cardiovasc Magn Reson 2014;16:9. doi:10.1186/1532-429X-16-9

17 Guiney TE, Davies MJ, Parker DJ, et al. The aetiology and course of isolated severe aortic regurgitation: a clinical, pathological, and echocardiographic study. British heart journal 1987;58:358-68.

18 Seder JD, Burke JF, Pauletto FJ. Prevalence of aortic regurgitation by color flow Doppler in relation to aortic root size. J Am Soc Echocardiogr 1990;3:316-9.

19 Roman MJ, Devereux RB, Niles NW, et al. Aortic root dilatation as a cause of isolated, severe aortic regurgitation. Prevalence, clinical and echocardiographic patterns, and relation to left ventricular hypertrophy and function. Ann Intern Med 1987;106:800-7.

20 Singh JP, Evans JC, Levy D, et al. Prevalence and clinical determinants of mitral, tricuspid, and aortic regurgitation (the Framingham Heart Study). AJC 1999;83:897-902.

21 Palmieri V, Bella JN, Arnett DK, et al. Associations of aortic and mitral regurgitation with body composition and myocardial energy expenditure in adults with hypertension: the Hypertension Genetic Epidemiology Network study. American heart journal 2003;145:1071-7. doi:10.1016/S0002-8703(03)00099-1 
Table 1. Characteristics of the study participants of the Cardiovascular Risk in Young Finns Study in middle age (measured in 2011). Values are presented as mean (SD) for continuous variables and n (\%) for categorical variables.

Descriptive Variables $\mathrm{N}=1950$

Age, years (range 34-49 years)

Males

$894(45.8 \%)$

Diabetic (data missing in $n=15$ )

Daily Smokers (\%) (data missing in $n=100$ )

Body-Mass Index, $\mathrm{kg} / \mathrm{m}^{2}$

Body Surface Area, $\mathrm{m}^{2}$

Overweight with body-mass index 25-30 (\%)

$704(36.1)$

Obese with body-mass index >30 (\%)

Systolic blood pressure, $\mathrm{mmHg}$

$118.9(14.1)$

Diastolic blood pressure, $\mathrm{mmHg}$

$74.8(10.5)$

Use of blood pressure lowering medication (data missing in $\mathrm{n}=100$ )

$184(10.0)$

Metabolic disorder According to National Cholesterol Education

Program (NCEP) criteria (\%) (data missing in $n=100)$

$412(21.4 \%)$

Aortic sinus diameter, cm (measured at Sinuses of Valsalva)

$3.21(0.35)$

Aortic sinus diameter indexed to Body Surface Area, $\mathrm{cm} / \mathrm{m}^{2}$

$1.69(0.19)$ 


\section{Baseline clinical examination in year 1980}

$\mathrm{n}=3,596(83 \%$ of invitees $)$

Randomly selected children and adolescents from six age cohorts: $3,6,9,12,15$ and 18 years of age

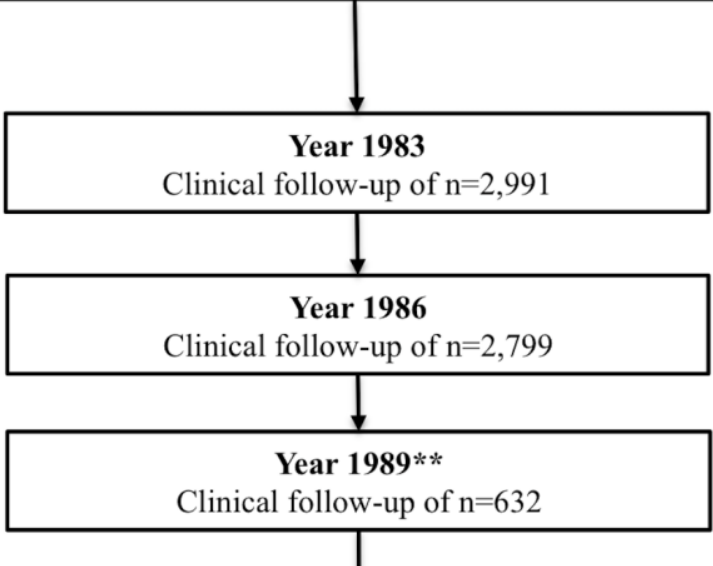

Year $1992 * *$

Clinical follow-up of $n=891$

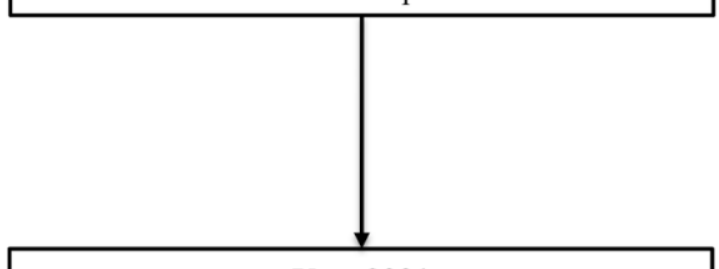

Year 2001

Clinical follow-up of $\mathrm{n}=2,276$

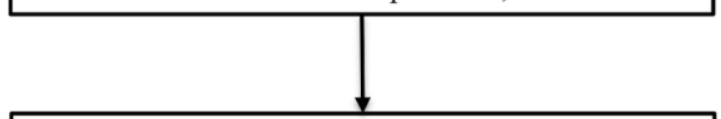

Year 2007

Clinical follow-up of $\mathrm{n}=2,172$

\section{Last clinical follow-up in year 2011}

Transthoracic ultrasound data available $n=1,950$ subjects with full data of body anthropometry from childhood to middle age*

Overall participation was $n=2063$ of which $n=94$ subjects excluded due to missing ultrasound data and additional $n=19$ due to other missing data.

Figure 1. Flow-chart of the longitudinal follow-up protocol of the Cardiovascular Risk in Young Finns Study. Anthropometric data was recorded at three-year intervals from childhood to middle age.

*Missing values for body size (height and weight) during early life (years 3-34) were estimated calculating subject-specific development curves for body weight and height were by mixed model regression splines using results from repeated measurements of the study

**Participants invited from only one of the five geographical areas (versus five in the entire cohort) 


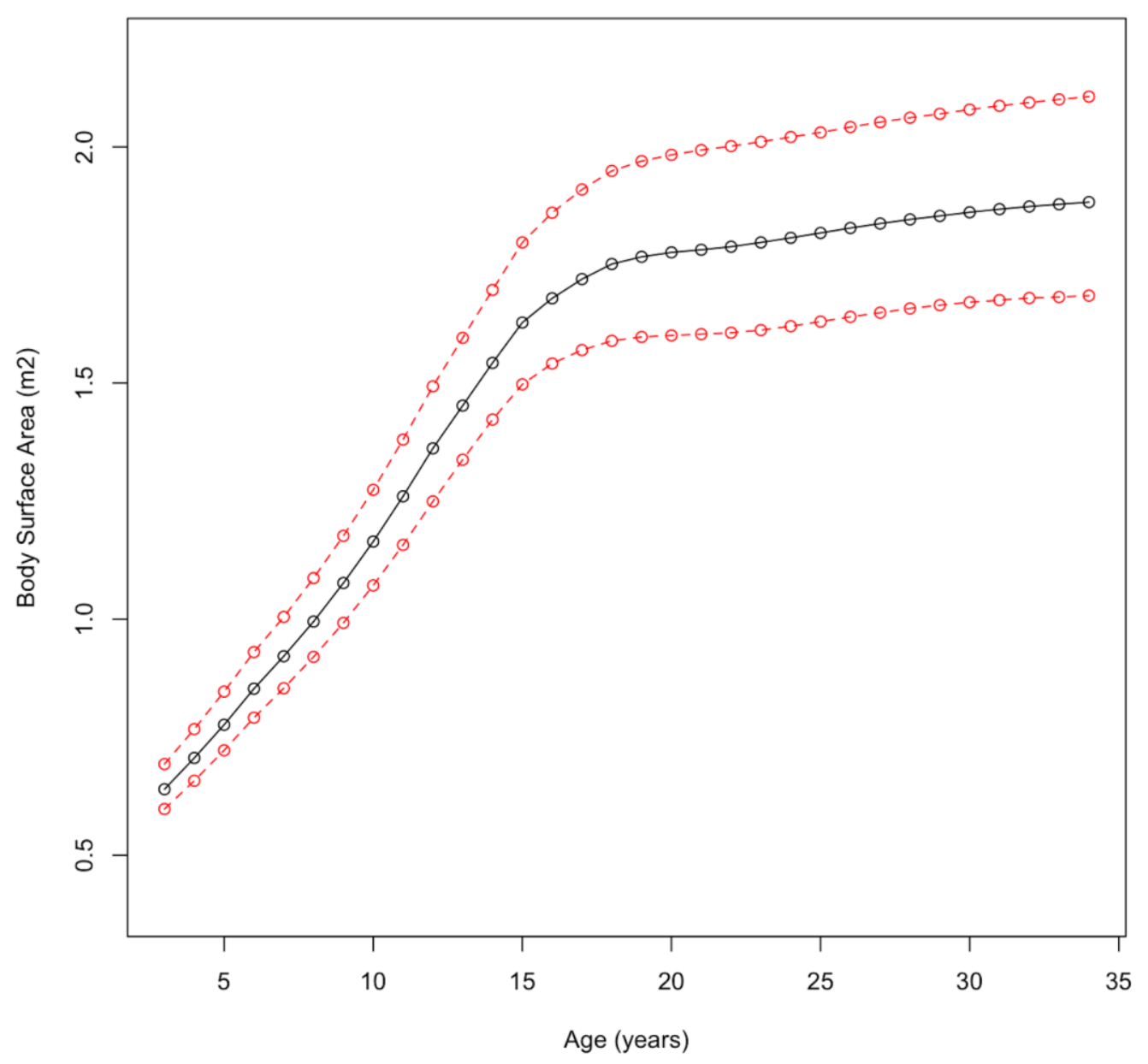

Figure 2. The development of mean body surface area in the Cardiovascular Risk in Young Finns Study population and the lower and upper limits of its standard deviation. 


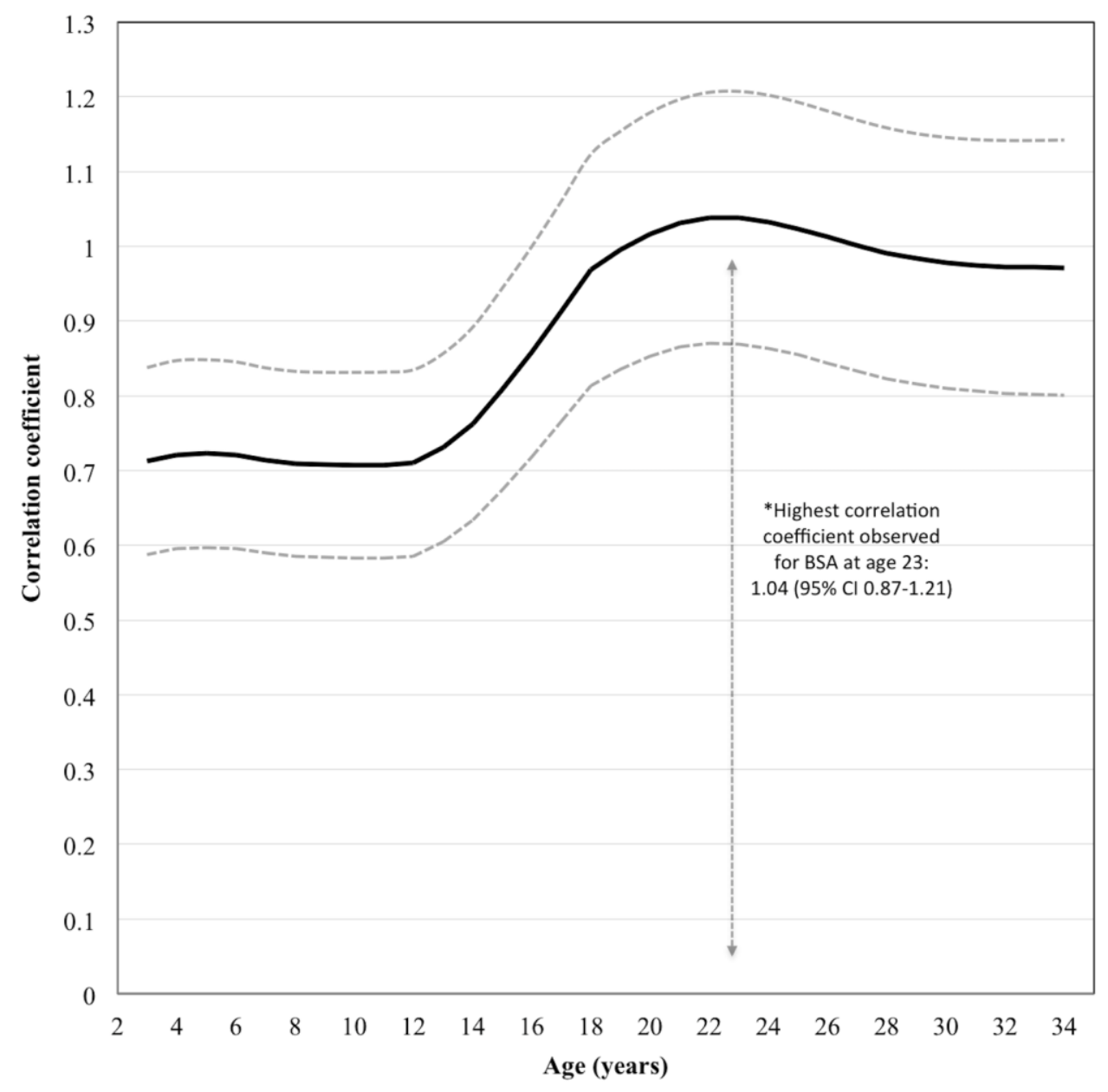

Figure 3. Curve of the association between body surface $\left(\mathrm{m}^{2}\right)$ area in different ages and aortic sinus size measured in middle age (34-49 years). The magnitude of the association in different ages (X-axis) is depicted by a continuous smoothed curve of regression coefficients (with 95\% confidence intervals) (values in Y-axis). The coefficient curve depicts the difference in aortic size $(\mathrm{mm})$ corresponding to one standard deviation increase in BSA in each age. The analysis was adjusted with age, sex and diastolic blood pressure in middle age. 

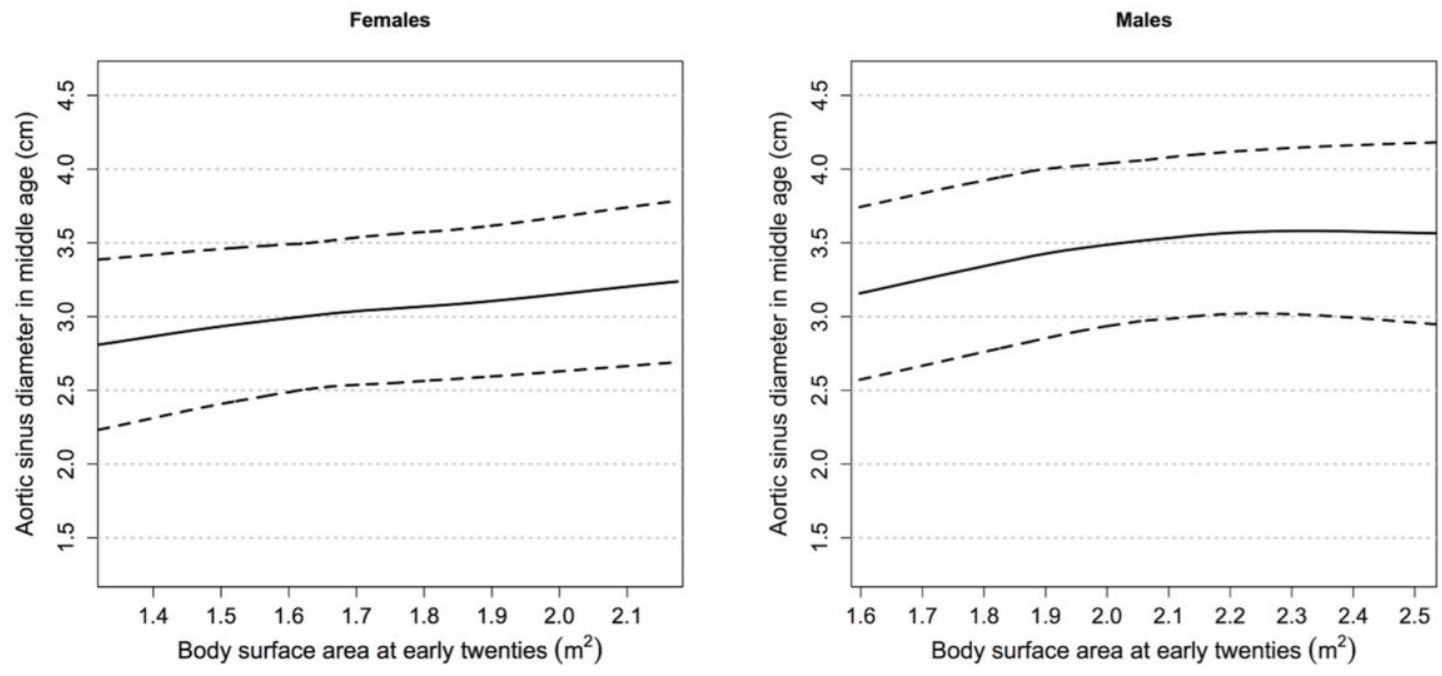

Figure 4. Aortic sinus diameter in middle age (y-axis) by BSA in early twenties (x-axis). The graphs illustrate smoothed mean response curves (solid line) for aortic sinus diameter in reference to BSA for both sexes. Dashed lines represent $95 \%$ intervals for the upper and lower limits for normal aortic sinus diameter. Response curves were reproduced by fitting a smoothed LOESS curve (Local weighted Scatterplot Smoothing by local regression) with 95\% prediction intervals. 